\title{
Traiettorie tecniche e Metodologia QWERTY: come si applicano alle innovazioni farmaceutiche
}

Marina Altagracia§, Mario Cimoli@, Jaime Kravzov®, Rafael Castillo¥, Ofelia Espejo¥, Albert I. Wertheimero

Articolo tratto dal Journal of Research in Pharmaceutical Economics, Vol. 10(1) 2000

E 2000 by The Haworth Press, Inc.

\begin{abstract}
Hypertension is a problem that has a social component and a biological one. The history of the biological solutions has been an increase in research activities and an increase in the number of new products introduced in the pharmaceutical market and adopted as usual therapies.

This article analyzes each of the main innovations in the treatment of moderate arterial hypertension and their competitive inter-relationships using a conceptual frame based on the recent theory of innovation. These innovations compete with each other for a market, similar to what happens with other areas and products. The concept of trajectories and the analysis of the competition process among them has not previously been applied to a pharmaceutical market and offers an exciting new avenue for insurance organizations, government health agencies, provider groups, and manufacturers to analyze trends and events in an organized and consistent framework.

Learning by using, pharmacological interrelatedness, and pharma-institutional networks are the main analytical tools used to explain the competing processes among the different trajectories considered.
\end{abstract}

Farmeconomia e percorsi terapeutici 2001; 2 (1): 19-36

\section{INTRODUZIONE}

La storia recente evidenzia come l'ipertensione sia un problema in costante crescita che pesa in modo rilevante sulla società. L'ipertensione è un problema che presenta una componente sociale e ed una biologica. Le scienze sociali hanno tentato di dare una soluzione al primo aspetto, mentre la ricerca di base e l'industria farmaceutica hanno cercato di risolvere il problema biologico dell'ipertensione mediante vari tipi di medicamenti.

Lo sviluppo di soluzioni biologiche contro l'ipertensione è stato attuato con l'incremento della ricerca che ha consentito di introdurre sul mercato un numero crescente di nuovi farmaci adottati via via come terapie standard. I laboratori delle più grandi industrie farmaceutiche che portano avanti progetti di ricerca e sviluppo (R\&D, Research \& Development) dedicano gran parte dei loro sforzi alla produzione di farmaci antiipertensivi, mentre altri laboratori più piccoli sono entrati in questo mercato nell'ultima decade.

Nel caso di innovazioni di prodotto (come i "me too" o analoghi), lo stadio finale del processo che va dalla ricerca scientifica alla produzione si ha quando l'articolo viene prodotto e venduto. In questo stadio finale i mercati operano nuovamente come ambienti selettivi. Oc- corre sottolineare che questa "selezione finale" ha una natura differente rispetto a quella degli stadi precedenti del processo. Nei percorsi tecnologici alcuni tipi di indicatori economici operavano come strumenti per scegliere le priorità tra un gran numero di possibili alternative tecnologiche. Qui invece il mercato opera come strumento di selezione a posteriori generalmente tra una gamma di prodotti già definiti dai modelli di una vasta tecnologia scelti dal versante dell' offerta.

In particolare, negli ultimi 50 anni, sono stati introdotti sul mercato molti nuovi prodotti sintetici (principi attivi) e ognuno di questi è stato concepito e presentato come una innovazione. In questo campo quindi, ogni novità compete con le altre per un mercato, così come accade per altre aree e prodotti. Come esempi consideriamo un veicolo, che può essere spinto da un motore a vapore, a scoppio o elettrico,benzina o elettricità; i metodi per la produzione di energia solare e nucleare; l'evoluzione dell'impostazione della tastiera della macchina da scrivere (QWERTY); la competizione, in elettronica, tra valvole, transistor e i più recenti circuiti integrati (1-3).

Questo articolo analizza tutte le principali innovazioni farmacologiche indicate per il trattamento dell'ipertensione arteriosa lieve o moderata e le reciproche relazioni di competizione,
§ Ricercatore e Professore dell'Universidad Autónoma Metropolitana-Xochimilco (UAM-

$X)$, Messico

(C) Medico, Professore dell'Università di Venezia e Docente Ospite presso la UAM-X (B) Dottore Honoris Causa presso la Puebla University, Professore alla UAM-X $¥$ Medico e Professore alla Universidad Nacional Autónoma de Mexico (UNAM) ๙ Direttore della Divisione ricerca e gestione delle risorse Merck \& Co., Inc., West Point, PA 19486 
utilizzando un modello concettuale basato sulla recente teoria dell'innovazione. In dettaglio, il razionale di questo lavoro si fonda su due elementi principali:

a) l'idea che l'insieme di farmaci innovativi che condividono un fondamentale meccanismo d'azione (classe dei farmaci antiipertensivi) possa essere rappresentato come una serie di differenti traiettorie in competizione tra di loro per l'acquisizione di un mercato;

b) il ruolo giocato dal mercato stesso e da altri meccanismi, come l'acquisizione di conoscenza dovuta all'uso, le economie di scala, le interrelazioni tecnologiche e le reti istituzionali.

Per collocare le diverse innovazioni entro le differenti soluzioni offerte, i concetti di paradigmi e di traiettorie tecnologiche vengono usati in relazione ai prodotti farmaceutici $(4,5)$. Questi concetti, già introdotti nel contesto degli studi di economia industriale, ci permetteranno di analizzare i differenti principi attivi innovativi come traiettorie che competono per un mercato che li adotti.

Le innovazioni farmaceutiche prese in considerazione sono riferite al mercato del Messico. In questo Paese il mercato farmaceutico è caratterizzato da una elevata quota di automedicazione e per una vasta gamma di medicinali non è richiesta la ricetta medica. Perciò, una volta registrati presso il Ministero della Sanità, i prodotti medicinali innovativi sono liberi di competere tra loro sul mercato. Tuttavia, la libertà di scelta di un farmaco piuttosto di un altro non significa necessariamente che il prodotto farmaceutico che domina il mercato sia il più efficiente sotto il profilo terapeutico e tecnologico.

Quando si prospetta una nuova opportunità economica di solito esistono diversi modi per realizzarla. In epoca moderna, ad esempio, l'energia nucleare può essere generata da reattori ad acqua leggera (raffreddati a gas) o ad acqua pesante (raffreddati a sodio) e l'energia solare può essere prodotta con tecnologie al silicio-cristallino o al silicio-amorfo.

Analogamente l'ipertensione può essere controllata con trattamenti farmacologici o non farmacologici. Una terapia farmacologica può essere attuata con farmaci di sintesi che abbiano come principale azione quella diuretica oppure qualche altro meccanismo d'azione, come nel caso dei vasodilatatori o dei calcioantagonisti. In ogni caso, possiamo immaginare che questi metodi o tecnologie siano "in competizione" per un "mercato" di utenti (6).

In questo contesto, cercheremo di identificare quali siano i fattori e come contribuiscano a spiegare la competizione tra le diverse traiettorie (5). In particolare, nell'ambito della farmacoeconomia si possono riscontrare e definire alcuni fattori come l'apprendimento derivante dall'uso del prodotto, la produzione in economie di scala, le interrelazioni tecnologiche, le esternalità di rete e l'incremento dei proventi dovuto all'informazione.

\section{Apprendere con l'uso}

"Spesso una tecnologia più viene adottata, più viene utilizzata e più viene conosciuta, quindi più si sviluppa e migliora. Ad esempio, il progetto di un nuovo aereo linea, come il DC-8, migliora notevolmente le caratteristiche che riguardano il carico pagante, la capienza passeggeri, l'efficienza del motore e l'aereodinamicità, quando viene adottato e utilizzato come aereo di linea" (7).

\section{La produzione in economie di scala}

Solitamente, quando una tecnologia viene riversata in un prodotto, come nel caso della tecnologia Polaroid, il costo del prodotto diminuisce con l'aumentare dell'unità prodotte. Perciò il prezzo della tecnologia può diventare più attraente con l'aumentare degli utenti.

\section{Le interrelazioni tecnologiche}

Spesso, quando una tecnologia si diffonde, diventano parte integrante della sua infrastruttura un certo numero di altre tecnologie correlate ed un certo numero di altri prodotti (8). Per esempio, intorno alla tecnologia dei carburanti si è sviluppata un'imponente infrastruttura di raffinerie, stazioni di rifornimento e industrie di componentistica d'automobile che da essa dipendono. Questo costituisce un vantaggio perchè altre tecnologie meno diffuse che volessero conquistare una fetta di mercato, soffrirebbero della mancanza di una tale rete di infrastrutture ed, anzi, potrebbero dover smantellare in parte le infrastrutture esistenti.

\section{Le esternalità di rete}

In genere una tecnologia offre maggiori vantaggi a coloro che la adottano se questi fanno parte di una rete più ampia (9). Il formato video VHS ne è un ottimo esempio: grazie alla vasta diffusione, gli utenti del formato VHS possono beneficiare della grande disponibilità e varietà dei prodotti registrati in VHS.

\section{L'incremento di proventi indotto dall'infor- mazione}

Spesso una tecnologia adottata su larga scala presenta il vantaggio di essere ben conosciuta e meglio compresa. Chi non vuole affrontare rischi preferisce scegliere una tecnologia il cui uso sia ampiamente diffuso. Naturalmente, in ogni specifica tecnologia possono essere presenti uno o più fattori capaci di 
favorine la diffusione. Raramente abbiamo un solo elemento capace di incrementare i proventi che derivano dalla diffusione della tecnologia (6).

IPERTENSIONE: UN PROBLEMA SOCIALE E LE SUE SOLUZIONI FARMACEUTICHE

L'ipertensione è uno dei maggiori problemi di salute pubblica in tutto il mondo e rappresenta il principale fattore di rischio di morte prematura e di inabilità. L'ipertensione è la malattia cardiovascolare più comune $(10,11)$. Il costo individuale del trattamento farmacologico può mettere in crisi il bilancio familiare del paziente e varia moltissimo tra i diversi tipi e categorie di prodotti farmaceutici, tra specialità brevettate e prodotti generici.

Secondo il National Poll of Chronic Diseases del Messico (dati del 1993), la prevalenza dell'ipertensione è del 24,6\%, ma se scartiamo i soggetti tra i 20 e i 30 anni e consideriamo solo la popolazione compresa tra 30 e 69 anni, la prevalenza sale al $29,1 \%$. Escludendo le persone sotto i 40 anni, la prevalenza raggiunge il $39 \%$ nella popolazione tra i 40 e i 69 anni. Nella fascia di età compresa tra i 50 e i 69 anni la prevalenza aumenta al 45\%. Il Messico conta una popolazione totale di 80 milioni di persone circa (12).

Una delle prime strategie messa a punto per la gestione dell'ipertensione fu quella di modificare il bilancio del sodio limitando il consumo di sale nella dieta. La modificazione farmacologica del bilancio del sodio fu realizzata praticamente negli anni '50 con lo sviluppo dei diuretici tiazidici somministrabili per via orale (10). La ditta Merck brevettò nel 1957 la clorotiazide, che fu il primo antipertensivo di sintesi, e nel 1962 l'idroclorotiazide (13). Questi medicamenti costituiscono un importante contributo al trattamento farmacologico dell'ipertensione e segnano l'inizio di questo tipo di terapia. I diuretici, probabilmente, costituiscono il più grande singolo contributo alla terapia farmacologica dell'ipertensione.

Possiamo classificare i farmaci antipertensivi in base al loro luogo e meccanismo d'azione. La pressione arteriosa è il prodotto della gittata cardiaca e della resistenza vascolare periferica e pertanto può essere $a b-$ bassata dall'azione di farmaci che agiscono o sulle resistenze periferiche o sulla gittata cardiaca o su entrambi i fattori. Grazie all'esistenza, o all'ipotesi, di differenti meccanismi d'azione, è stato possibile sviluppare una grande varietà di farmaci antipertensivi. Tuttavia, l'esatto meccanismo d'azione non è ancora completamente chiarito, e questo vale anche per farmaci utili ed utilizzati da molto tempo come i diuretici (10).
Negli anni '60 sono state introdotte nella pratica clinica (brevettate e registrate) alcune tra le principali classi o categorie di farmaci antipertensivi, tra cui gli antiadrenergici (inibitori del neurone periferico) ed $\mathrm{i}$ vasodilatatori (14-16). Esempi di queste due categorie sono, rispettivamente, la metildopa e l'idralazina.

Durante gli ultimi vent'anni (dal 1980 al 1997), l'introduzione di farmaci come i calcioantagonisti e gli ACE-inibitori ha contribuito a comprendere la fisiopatologia dell'ipertensione $(11,15-16)$. Molecole prototipo di queste due categorie sono il verapamil, la nifedipina ed il diltiazem, per quanto riguarda i calcio-antagonisti, ed il captopril per gli ACE-inibitori. In seguito sono stati sviluppati, approvati e introdotti nella pratica clinica come antipertensivi molti altri calcio- antagonisti ed ACE- inibitori (17).

La ricerca di base prosegue e l'industria farmaceutica è ancor sempre alla ricerca della soluzione ottimale: farmaci che siano efficaci come monoterapia nel 50\% dei casi ed assicurino il controllo della pressione arteriosa per 24 ore, richiedano una sola somministrazione al giorno e siano ben tollerati, abbiano effetti biochimici e metabolici favorevoli, riducano il danno d'organo, promuovano un buon livello di compliance, abbiano una bassa incidenza di reazioni avverse e permettano una buona qualità di vita.

Se confrontiamo gli investimenti in R\&D delle ditte farmaceutiche e lo sviluppo di farmaci antipertensivi, notiamo che Roche, Merck, Ciba, Pfizer e Upjohn hanno brevettato molti farmaci antipertensivi. A partire dagli anni '50 queste industrie e i loro prodotti sono stati leader del mercato nazionale e internazionale. Le tre ditte che hanno fatto i maggiori investimenti in ricerca e sviluppo negli ultimi anni sono Glaxo-Wellcome (con 1,9 miliardi di dollari Usa nel 1996), Ciba/Sandoz (1,7 miliardi \$ Usa) e Roche (1,6 miliardi \$ Usa), seguite da Merck, Pfizer e Upjohn (18).

Ogni nuovo principio attivo viene considerat un' innovazione. L'idea che qui proponiamo è che studiando come ognuna delle categorie o classi terapeutiche ha affrontato la competizione sul mercato si possa correlare questo con la soluzione al problema dell'ipertensione essenziale. È anche interessante esaminare la competizione tra molecole prototipo ed analoghi. I vari gruppi terapeutici e i loro principi attivi aggrediscono il problema e forniscono soluzioni mediante differenti meccanismi d'azione ma ottenendo lo stesso risultato finale (una riduzione della pressione arteriosa), e si evidenzia una dinamica di competizione tra i diversi gruppi e tra i diversi prodotti (principi attivi) che li compongono. 
PARADIGMI SOCIO-TECNOLOGICI, TRAIETTORIE ED ECONOMIA DELLA METODOLOGIA QWERTY

Concetto fondamentale, necessario adesso per descrivere la natura evolutiva dell'innovazione a livello microeconomico, è il paradigma tecnologico (5). Adattando la nozione di paradigma formulata da Kuhn nell' ambito delle scienze filosofiche, Dosi ha definito un paradigma tecnologico come "un modello di soluzione per problemi tecnoeconomici selezionati, basato su principi altamente selezionati derivati dalle scienze naturali, unitamente a regole specifiche finalizzate ad acquisire nuove conoscenze ed a salvaguardarle, quando possibile, dalla rapida diffusione ai competitori" (19). Inoltre "una soluzione innovativa di certe problematiche richiede la scoperta e la creazione, visto che nessun algoritmo generale capace di generare automaticamente la soluzione può essere ricavato dall'informazione sul problema". Le caratteristiche di dipendenza e contingenza derivano dal fatto che "la soluzione di problemi tecnologici coinvolge l'utilizzo di informazioni dedotte da precedenti esperienze e da conoscenze formali e richiede, comunque, anche costanti abilità specifiche da parte degli inventori" (19). Perciò il risultato del processo di ricerca sarà determinato dalla storia dell'inventore, dalla conoscenza formale disponibile e dall' abilità dell' inventore.

I paradigmi in genere definiscono anche modelli base di manufatti e sistemi, che nel tempo vengono progressivamente modificati e migliorati. Questi prodotti basilari possono anche essere descritti secondo alcune caratteristiche tecnologiche ed economiche fondamentali. Ad esempio, nel caso di un aeroplano, questi attributi di base vengono descritti non solo in termini di risorse e di costi di produzione, ma anche sulla base di alcuni elementi tecnologici salienti quali la portanza delle ali, il peso al decollo, la velocità, la distanza che può coprire, etc. L'aspetto interessante è che il progresso tecnico sembra mostrare modelli di invarianza per quanto riguarda queste caratteristiche di prodotto. Esempi simili di invarianza tecnologica si possono trovare nel campo dei semiconduttori, delle attrezzature agricole, delle automobili e in poche altre aree di studi microtecnologici.

Paradigmi si trovano generalmente in sistemi tecnologici di maggiori dimensioni ed in ancora più vasti sistemi dei più grandi settori economici produttivi ed innovativi. Queste caratteristiche evolutive del processo di modificatione della tecnica sono viste in una prospettiva complementare come responsabili della manifestazione di quel fenomeno che è stato chia- mato "lock-in (chiusura) da eventi storici".

Questo concetto suggerisce una visione del processo di selezione e di adozione delle tecnologie come dominato da dipendenza da percorsi, da imprevedibilità, da inflessibilità (maggiore è la diffusione di una particolare tecnologia, minori chance di affermazione avranno altre tecnologie) e da possibilità di selezionare tecnologie inferiori (un esempio di quest'ultima situazione è l'adozione dei reattori ad acqua leggera al posto di quelli raffreddati a gas, che adesso sono considerati inferiori). L'approccio "lock-in" comporta alcune implicazioni, tra cui il fatto che la storia di un'innovazione e le sue interazioni con i fenomeni di apprendimento mediante l'uso, di esternalità di rete, di produzione secondo economie di scala, di incremento dei proventi mediante l'informazione assumono un ruolo fondamentale per capire i meccanismi di competizione con altre tecnologie e per comprendere se l'adozione possa sostituire o precludere l'adozione di altre tecnologie $(1,3,20-21)$.

Per quanto riguarda l'ipertensione arteriosa essenziale, è noto che essa rappresenta un problema sociale e tecnologico definito da valori di pressione sistolica e diastolica superiori, rispettivamente, a 140 e $90 \mathrm{~mm}$ di mercurio. In questo contesto si considera normale una pressione arteriosa uguale o inferiore a $140 / 90 \mathrm{~mm}$ di mercurio. Si considerano ipertesi lievi i pazienti che hanno valori di pressione diastolica compresi tra 90 e $104 \mathrm{~mm}$ di mercurio, mentre sono considerati ipertesi moderati quelli che hanno valori compresi tra 105 e 114 mm di mercurio (10). In un tale contesto ci apprestiamo a introdurre il concetto del paradigma socio-tecnologico nel campo farmaceutico. Si definisce paradigma l'insieme di soluzioni farmacologiche finalizzati a rispondere ad un problema sociotecnologico (ipertensione). In altre parole, si tratta di farmaci indirizzati a risolvere un problema che si evidenzia in termini di parametri sociali e biologici, nell' ambito del quale la ricerca di base e l' industria chimico-farmaceutica svolgono un ruolo preminente.

Il concetto di traiettorie tecnologiche è associato alla progressiva realizzazione di opportunità innovative correlate ad ogni singolo paradigma, che , in linea di principio, può essere misurato secondo i termini dei cambiamenti che si verificano nelle caratteristiche tecnoeconomiche fondamentali dei manufatti e nel processo di produzione. Nelson e Winter hanno definito traiettorie naturali del progresso tecnico quei percorsi che contribuiscono a disegnare la direzione lunga la quale si dirigono le attività rivolte alla soluzione di problemi e che possiedono in sé uno slancio particolare; in questo senso, una traiettoria rappresenta la 
normale attività di problem-solving determinata da un paradigma $(19,22)$. Il nucleo centrale delle idee che stanno alla base di questo concetto di traiettoria si può riassumere nei seguenti punti: primo, ogni particolare insieme di conoscenze (ad esempio, ogni paradigma) plasma e vincola la velocità e la direzione del cambiamento tecnologico indipendentemente dagli stimoli del mercato e, in secondo luogo, come conseguenza, si possono osservare regolarità e invarianze nel modello di mutamento tecnologico, le quali persistono inalterate in differenti condizioni di mercato (ad esempio in condizioni di prezzi relativi diversi) mentre la loro rottura avviene in relazione a radicali cambiamenti nella conoscenza di base (nel paradigma).

Nel corso degli ultimi 50 anni sono state offerte diverse specifiche soluzioni per risolvere il problema dell'ipertensione ed ognuna di loro si presenta, ogni volta, come una traiettoria. Ognuna di queste traiettorie è apparsa sul mercato come una proposta innovativa e noi osiamo sperare che intorno a ciascuna di loro sia stata costruita una maggiore efficienza terapeutica nel trattamento dell'ipertensione. Poichè ciascuna di queste proposte ha una diversa e sconosciuta via fisiologica, fin dal momento della loro comparsa ognuna è stata considerata un'innovazione ed un importante contributo farmacologico.

In questo arco di tempo abbiamo individuato cinque traiettorie per il trattamento dell'ipertensione essenziale (Tabella 1). Ora descriveremo queste traiettorie, indicando per ciascuna $i$ meccanismi generali della loro azione biologica.

La traiettoria $\mathrm{T}_{1}$ è quella che riguarda $\mathrm{i}$ diuretici tiazidici, sintetizzati e brevettati nel 1950 (13). I diuretici riducono il volume plasmatico, bloccando il riassorbimento tubulare di acqua e sodio e agendo sulla parete dei vasi arteriolari. Tutti i farmaci di questa classe hanno effetti simili sulla riduzione della pressione arteriosa ed anche simili effetti collaterali. I diuretici tiazidici possono causare ipopotassiemia, iperglicemia, eritemi e altre reazioni allergiche, disfunzioni sessuali e spossatezza $(10,23)$.

La traiettoria $\mathrm{T}_{2}$ comprende $\mathrm{i}$ simpatolitici periferici (farmaci antiadrenergici), sintetizzati e brevettati a partire dagli anni 1963-1967 (i primi ) (13). Questi farmaci riducono la frequenza e il lavoro cardiaco, riducono l'attività reninica plasmatica e possono far aumentare le resistenze periferiche. Il loro meccanismo d'azione non è ancora chiaro (10). I farmaci antiadrenergici possono causare affaticamento, depressione, bradicardia, insufficienza cardiaca congestizia, broncospasmo, cefalea, spossatezza e sonnolenza (23).
La traiettoria $\mathrm{T}_{3}$ è quella dei farmaci vasodilatatori brevettati tra il 1952 e il 1979 (Tabella 1) (13). La loro azione antipertensiva è dovuta ad un effetto vasodilatatore sui vasi sanguigni ed anche ad un effetto rilassante sui muscoli vascolari. Spesso i vasodilatatori $\left(\mathrm{T}_{3}\right)$ producono tachicardia $(10,23)$.

La traiettoria $T_{4}$, è quella che riguarda $\mathrm{i}$ calcioantagonisti sviluppati dal 1987 ad oggi (17). Essi sono stati introdotti sul mercato come antipertensivi poco dopo gli ACE-inibitori (gruppo $\mathrm{T}_{5}$ ). I calcioantagonisti riducono la pressione arteriosa, rilassano i muscoli lisci arteriolari e migliorano la resistenza vascolare periferica. Essi riducono il flusso transmembrana del calcio e, di conseguenza, riducono la quantità totale di calcio che raggiunge i siti intracellulari (10). Alcuni di questi farmaci, prima di essere formalmente approvati come antipertensivi, venivano già usati in alcuni paesi (fuori dagli USA) per altre indicazioni.

Il Verapamil $\left(\mathrm{T}_{4}\right)$ e altri calcioantagonisti possono causare una forte stipsi. Il Diltiazem può provocare vertigini, cefalee, ecc. Le diidropiridine (nifedipina, nitrendipina, amlodipine, ecc.) possono invece causare edemi (23).

La traiettoria $\mathrm{T}_{5}$ è quella della classe degli ACE-inibitori. Il captopril, sintetizzato nel 1981, è stato il prototipo di questa classe ed ancora oggi gli scienziati continuano a modificarne la struttura per inventare analoghi il cui numero continua ad aumentare, alimentando questa classe farmacologica e quelle che sono considerate sub-traiettorie (sotto gruppi di principi attivi analoghi appartenenti ad una traiettoria) (vedi Tabella 2). Si ritiene che gli ACE-inibitori agiscano fondamentalmente attraverso la soppressione del sistema renina-angiotensinaaldosterone, anche se non è stata ancora descritta una correlazione consistente tra livelli di renina e la risposta a questi farmaci (10).

Una tosse insensibile ai farmaci insorge nel 5-20\% dei pazienti trattati con ACE-inibitori (24). L'angioedema, che può anche rivelarsi fatale, si manifesta in una percentuale che va dallo $0,1 \%$ allo $0,2 \%$ dei pazienti curati con ACEinibitori, e richiede l'immediata sospensione della terapia (25). Questo tipo di farmaci non dovrebbe essere usato in gravidanza poiché può causare gravi danni e anche la morte del feto (26).

Le traiettorie $\mathrm{T}_{1}-\mathrm{T}_{5}$ sono presentate sotto forma di tabella, con l'indicazione dei farmaci più comuni per ognuna (principi attivi o molecole biologicamente attive), dei brevetti, dei laboratori che li hanno ottenuti e della data di approvazione da parte della Food and Drug Administration degli Stati Uniti (Tabella 1). 


\begin{tabular}{|c|c|c|c|c|}
\hline & & Brevetto & Laboratorio & $\begin{array}{c}\text { Data } \\
\text { approvazione } \\
\text { US FDA }\end{array}$ \\
\hline & $T_{1}$ Diuretici & & & \\
\hline & 1 Clorotiazide & $\begin{array}{l}\text { US pat. } 2,809,194 \& \\
2,937,169(1957 \& 1960)\end{array}$ & Merck \& Co. & 1958 \\
\hline & 2 Bendroflumetiazile $^{\mathrm{A}}$ & US pat. $3,265,573$ (1966) & Squibb & 1959 \\
\hline & 3 Idroclorotiazide $^{\mathrm{A}}$ & US pat. 3,025,292 (1962) & Merck \& Co. & 1959 \\
\hline & 4 Idroflumetazide & US pat. $3,254,076$ (1966) & $\begin{array}{l}\text { Lövens Kemiske } \\
\text { Fabrik }\end{array}$ & 1959 \\
\hline & 5 Benzotiazide $^{\mathrm{B}}$ & US pat. $3,440,244$ (1962) & Pfizer & 1960 \\
\hline & 6 Meticlotiazide & $\begin{array}{l}\text { Close et al. J. Am. Soc. } \\
82,1132 \text { (1960) }\end{array}$ & ------- & 1960 \\
\hline & 7 Triclormetiazide $^{\mathrm{B}}$ & $\begin{array}{l}\text { deStevens et al. Experentia, } \\
16,113(1960)\end{array}$ & ------- & 1960 \\
\hline & 8 Politiazide & US pat. $3,009,911(1961)$ & Pfizer & 1961 \\
\hline & 9 Ciclotiazide $^{\mathrm{C}}$ & US pat. 3,275,625 (1966) & Boehringer, Ing. & 1961 \\
\hline & 10 Clortalidone $^{\mathrm{D}}$ & US pat. 3,051,904 (1962) & Ciba Geigy & 1960 \\
\hline & 11 Chinetazone $^{\mathrm{B}}$ & US pat. $2,976,289$ (1961) & Cyanamid & 1962 \\
\hline & 12 Metolazone & US pat. 3,360,518 (1967) & Wallace \& Tiernan & 1974 \\
\hline & 13 Ticrinafen ${ }^{B}$ & US pat. $3,758,506(1971)$ & C.E.R.P.H.A. & 1979 \\
\hline & 14 Furosemide A, E & US pat. $3,058,882$ (1962) & Hoechst & 1966 \\
\hline & 15 Acido Etacrinico $^{\mathrm{B}}$ & US pat. $3,255,241$ (1962) & Merck \& Co & 1967 \\
\hline & 16 Bumetanide $^{P}$ & US pat. $3,806,534$ (1970) & $\begin{array}{l}\text { Lövens Kemiske } \\
\text { Fabrik }\end{array}$ & 1983 \\
\hline & 17 Indapamide $^{\mathrm{B}}$ & US pat. $3,565,911$ (1969-71) & $\begin{array}{l}\text { Sci. Union et } \\
\text { Acie, Soc. Franc. } \\
\text { Recherche Med. }\end{array}$ & 1983 \\
\hline & 18 Spironolattone $\mathrm{A}, \mathrm{F}$ & US pat. $3,013,012$ (1961) & Searle & 1959 \\
\hline & 19 Triamterene $^{\mathrm{A}}$ & US pat. $3,081,230$ (1963) & SK \& F & 1964 \\
\hline & 20 Amiloride $^{\mathrm{A}}$ & Belg. pat. 639,386 (1964) & Merck \& Co. & 1981 \\
\hline & \begin{tabular}{|ll}
$T_{2}$ & Adrenergici \\
\end{tabular} & & & \\
\hline & 1 Metildopa ${ }^{\mathrm{A}}$ & US pat. $2,868,818(1959)$ & Merck \& Co. & 1963 \\
\hline & 2 Clonidina $\mathrm{A}, \mathrm{G}$ & US pat. $3,202,660(1965)$ & Boehringer Ing. & 1974 \\
\hline & 3 Guanabenz $^{\text {B }}$ & $\begin{array}{l}\text { Brit. Pat. 1,019,120 (1966)/ Ger. } \\
\text { Pat. 1,804,634 (1969) }\end{array}$ & Shell/Sandoz & 1982 \\
\hline & 4 Alseroxilon $^{\mathrm{H}}$ & ------- & ------- & 1953 \\
\hline & 5 Criptenamina $^{\mathrm{B}}$ & US pat. 2,789,977 (1957) & Irwin, Neisler & 1953 \\
\hline & 6 Reserpina $^{\mathrm{A}}$ & US pat. 2,833,771 (1958) & Ciba Geigy & 1953 \\
\hline & 7 Rescinnamina $^{\mathrm{A}}$ & US pat. $2,876,228$ (1959) & Pfizer & 1956 \\
\hline & 8 Deserpidina $^{\mathrm{B}}$ & US pat. $2,982,769$ (1961) & Ciba Geigy & 1957 \\
\hline & 9 Guanetidina ${ }^{A}$ & US pat. $2,928,829$ (1960) & Ciba Geigy & 1960 \\
\hline & 10 Guanadrel $^{\mathrm{B}}$ & US pat. $3,547,951$ (1968) & Cutter & 1983 \\
\hline & 11 Prasozin A, I & US pat. $3,511,836(1969)$ & Pfizer & 1976 \\
\hline & 12 Terazosin $^{\mathrm{A}}$ & US pat. 4,026,894 (1977) & Abbott & -------- \\
\hline & 13 Doxazosin $^{B}$ & US pat. $4,188,390$ (1979) & Pfizer & ------- \\
\hline 1 & 14 Urapidil $^{B}$ & US pat. $3,957,786$ (1971) & Byk-Gulden & ------- \\
\hline$T_{1}-T_{5}$ Traiettorie & 15 Propranololo A, L & US pat. 3,337,628 (1964) & ICI & 1967 \\
\hline dei farmaci per & 16 Metoprololo $^{\mathrm{A}}$ & US pat. $3,873,600$ (1971) & AB Hässle & 1978 \\
\hline $\begin{array}{l}\text { l'ipertensione nella } \\
\text { storia. }\end{array}$ & 17 Nadololo $^{\mathrm{A}}$ & US pat. 3,395,267 (1973) & Squibb & 1979 \\
\hline
\end{tabular}




\begin{tabular}{|c|c|c|c|}
\hline 18 Atenololo $^{\mathrm{A}}$ & US pat. 3,663,607 (1970) & ICI & 1981 \\
\hline 19 Timololo $^{\mathrm{A}}$ & US pat. 3,655,663 (1972) & Frosst & 1981 \\
\hline 20 Pindololo $\mathrm{A}$ & $\begin{array}{l}\text { Swiss pat. 469,002 (1969) \& } \\
472,404(1969)\end{array}$ & Sandoz & 1981 \\
\hline 21 Betaxololo $^{\mathrm{B}}$ & US pat. 4,252,984 (1977) & Synthelabo & ------- \\
\hline 22 Penbutololo $^{\mathrm{B}}$ & US pat. 3,551,493 (1969) & Hoechst & -------- \\
\hline 23 Acebutololo $^{\mathrm{B}}$ & US pat. $3,857,952$ (1969) & May \& Baker & 1984 \\
\hline 24 Labetalolo $^{\mathrm{B}, \mathrm{M}}$ & US pat. 4,012,444 (1971) & Allen \& Hanburys & 1984 \\
\hline 25 Mecamilamina $^{\mathrm{B}, \mathrm{N}}$ & US pat. $2,831,027$ (1958) & Merck \& Co & 1956 \\
\hline 26 Pargilina $^{\mathrm{B}, \mathrm{O}}$ & US pat. $3,155,584$ (1962) & Abbott & 1963 \\
\hline \multicolumn{4}{|l|}{$\begin{array}{ll}\mathbf{T}_{3} & \text { Vasodilatatori }\end{array}$} \\
\hline 1 Idralazina $^{\mathrm{A}, \mathrm{P}}$ & US pat. 2,484,029 (1949) & Ciba-Geigy & 1952 \\
\hline 2 Minoxidil $\mathrm{B}$ & US pat. 3,382,247 (1967) & Upjohn & 1979 \\
\hline 3 Nitroglicerina $^{(\mathrm{N})}, \mathrm{A}, \mathrm{Q}$ & Subrero Ann 64,398 (1847) & ------- & ------- \\
\hline 4 Pinacidil B, R & US pat. 4,057,636 (1976) & Leo Pharm & 1989 \\
\hline \multicolumn{4}{|c|}{$\mathrm{T}_{4}$ Agenti bloccanti del canale calcio } \\
\hline 1 Diltiazem $^{\mathrm{A}, \mathrm{S}}$ & US pat. 3,562,257 (1969) & Tanabe Seiyaku & 1988 \\
\hline 2 Verapamil A, T & US pat. 3,261,859 (1962) & Knoll & 1988 \\
\hline $3 \quad$ Gallopamil $^{\mathrm{A}}$ & $\begin{array}{l}\text { US pat. 3,261,859 (1962) US } \\
\text { pat. } 4,115,432(1978)\end{array}$ & Knoll & ------ \\
\hline 4 Nifedipina $^{\mathrm{A}, \mathrm{U}}$ & US pat. 3,485,847 (1969) & Bayer & 1987 \\
\hline 5 Nicardipina $^{\mathrm{A}}$ & US pat. 3,985,758 (1974) & Yamanouchi & 1988 \\
\hline 6 Nimodipina $^{\mathrm{A}}$ & US pat. 3,799,934 (1972) & Bayer & ------ \\
\hline 7 Amlodipina $^{\mathrm{A}}$ & US pat. $4,572,909$ (1983) & Pfizer & 1992 \\
\hline 8 Felodipina $^{\mathrm{A}}$ & US pat. 4,264,611 (1981) & AB Hassle & 1991 \\
\hline 9 Nitrendipina $^{\mathrm{A}}$ & US pat. 3,799,934 (1974) & Bayer & 1987 \\
\hline 10 Isradipina $^{\mathrm{A}}$ & US pat. $4,466,972(1984)$ & Sandoz & 1990 \\
\hline \multicolumn{4}{|c|}{$\mathbf{T}_{5}$ Inibitori dell'enzima di conversione dell'angiotensina (ACE) } \\
\hline 1 Captopril A, V $^{2}$ & US pat. $4,046,889$ (1977) & Squibb & 1981 \\
\hline 2 Lisinopril $^{\text {A }}$ & $\begin{array}{l}\text { US pat. 4,374,829 (1983); US } \\
\text { pat. } 4,555,502(1985)\end{array}$ & Merck \& Co. & 1987 \\
\hline 3 Enalapril $^{\mathrm{A}}$ & US pat. 4,374,829 (1983) & Merck \& Co. & 1985 \\
\hline 4 Ramipril A $^{A}$ & US pat. $4,587,258(1986)$ & $\begin{array}{l}\text { Hoechst Schering } \\
\text { Corp. }\end{array}$ & 1991 \\
\hline $5 \quad$ Benazepril $^{\mathrm{A}}$ & US pat. 4,410,520 (1983) & Ciba Geigy & 1991 \\
\hline 6 Quinapril A $^{\mathrm{A}}$ & US pat. 4,344,949 (1982) & Warner-Lambert & 1991 \\
\hline $7 \quad$ Fosinopril A & US pat. 4,337,302 (1982) & Squibb & 1991 \\
\hline 8 Cilazapril $^{\mathrm{A}}$ & US pat. $4,512,924$ (1985) & Hoffmann-La Roche & 1992 \\
\hline 99 Trandolapril ${ }^{\text {в }}$ & US pat. 4,933,361 (1990) & Hoechst & 1997 \\
\hline 10 Perindopril $^{\mathrm{B}}$ & US pat. 4,508,729 (1985) & Adir-France & 1988 \\
\hline
\end{tabular}

Fonte: Merck Index 11th \& 12th Editions; The Medical Letter and the Chemical Abstract Patent Index.

Legenda Tabella 1:

A Products in Mexican market (only oral pharmaceutic presentations were included).

B Products not in the Mexican market.

C Thiazide agents are chemical analogs with the same pharmacologic action.

D Thiazide-like agents are chemically different from the thiazide diuretic but have similar pharmacologic action.

Looping-acting agents

Potassium-sparing agents

Central -receptor stimulator

Peripheral neuronal inhibitor 
I -receptor blockers

L -receptor blockers

M \& receptor blockers

N Ganglionic neural blockers

O Monoamine oxidase inhibitor

US FDA Calcium channel blockers only after 1980 were approved by US FDA for the treatment of hypertension

$\mathrm{P} \quad$ Vasodilators (general action) are chemically different with the same pharmacologic action.

Q Vein vasodilators

$\mathrm{R} \quad$ Potassium-sparing

S Diltiazem (calcium antagonist), verapamil, and nifedipine are chemically different with the same pharmacologic action

$\mathrm{T} \quad$ Verapamil (Calcium antagonist) are chemically different from diltiazem and nifedipine, but with the same pharmacologic action

$\mathrm{U} \quad$ Nifedipine (Calcium antagonist) are chemically different from diltiazem and verapamil analogs but with the same pharmacologic action

$\mathrm{V} \quad$ ACE inhibitors are chemical analogs with the same pharmacologic action.

Tabella 2

Inibitori ACE (traiettoria $\left.T_{5}\right) e$

Captoril come molecole prototipi, e loro analoghi (Sub-traiettorie $\left.T_{5,1} T_{5,8}\right)$.

\begin{tabular}{|lccc}
\hline $\begin{array}{l}\text { Nome prodotto } \\
\text { messicano }\end{array}$ & Composizione chimica & $\begin{array}{c}\text { Ditta } \\
\text { farmaceutica }\end{array}$ & $\begin{array}{c}\text { Data di introduzione sul } \\
\text { mercato messicano }\end{array}$ \\
\hline 1. Capotena & Captopril & Squibb & $9 / 82$ \\
\hline 2. Renitec & Enalapril & Merck Sharp \& Dohme & $4 / 86$ \\
\hline 3. Zestril & Lisinopril & Zeneca & $4 / 88$ \\
\hline 4. Prinivil & Lisinopril & Merck Sharp \& Dohme & $4 / 88$ \\
\hline 5. Glioten & Enalapril & Armstrong & $7 / 90$ \\
\hline 6. Ecapresan & Captopril & Mead Johnson & $9 / 90$ \\
\hline 7. Ecaten & Captopril & Fustery & $2 / 91$ \\
\hline 8. Acupril & Quinapril & Parke Davis & $5 / 91$ \\
\hline 9. Inhibace & Cilazapril & Roche & $5 / 91$ \\
\hline 10. Enaladil & Enalapril & Siegfried & $7 / 91$ \\
\hline 11. Lutensin & Benazepril & Ciba-Geigy & $9 / 91$ \\
\hline 12. Cardipril & Captopril & Liomont & $10 / 91$ \\
\hline 13. Monopril & Fosinopril & Mead Johnson & $10 / 91$ \\
\hline 14. Tritace & Ramipril & Hoechst & $10 / 92$ \\
\hline 15. Ramace & Ramipril & Astra & $11 / 92$ \\
\hline 16. Captral & Captopril & Silanes & $8 / 94$ \\
\hline 17. Ecapril & Captopril & Promeco & N.D. \\
\hline \hline 18. Lenpryl & Captopril & Galen & \\
\hline 19. Kenolan & Captopril & Kendrick & N. \\
\hline
\end{tabular}

Fonti:

Diccionario De Especialidades Farmacéuticas (PML). Mexican Directory of Pharmaceuticals, 1997. International Marketing Services México, 1997.

\section{EFFICIENZA TERAPEUTICA}

Il trattamento farmacologico è riservato a quei pazienti ipertesi che non riescono a mantenere la pressione arteriosa entro i limiti normali con mezzi non farmacologici. L'obiettivo di una terapia farmacologica è quello di ridurre la pressione diastolica sotto i 90 millimetri di mercurio (10).

Fin dal 1977 l'U.S. Joint National Committee on Detection, Evaluation and Treatment of High Blood Pressure (JNC) ha elaborato sei strategie per agevolare la scelta dei farmaci antipertensivi (14, 27-31). Queste raccomandazioni, che si basano sull' efficacia e sull'efficienza dei farmaci, hanno ottenuto il riconoscimento delle rispettive comunità scientifiche. La JNC non raccomanda singole specifiche molecole, ma classi terapeutiche di farmaci, ben sapendo che gli antipertensivi che appartengono ad una medesima classe (traiettoria per questo lavoro) hanno azioni terapeutiche uguali o simili nel trattamento dell'ipertensione lieve o moderata.

Da un punto di vista tecnologico le classi farmacologiche raccomandate dal JNC sono formate dall'identificazione delle traiettorie considerate: $\mathrm{i}$ diuretici (gruppo $\mathrm{T}_{1}$ ), gli antiadrenergici (gruppo $\mathrm{T}_{2}$ ), i vasodilatatori (gruppo $\mathrm{T}_{3}$ ), i calcioantagonisti (gruppo $\mathrm{T}_{4}$ ) e gli ACE-inibitori (gruppo $\mathrm{T}_{5}$ ). Queste categorie 
o classi farmacologiche sono state considerate come terapie di prima scelta per il trattamento dell'ipertensione arteriosa cronica e sono incluse in una strategia terapeutica (proposta dal JNC nel 1980 e nel 1992 e recentemente aggiornata con il sesto report del 1997) che consiste di cinque tappe e prende in considerazione i differenti livelli di complessità del trattamento e la resistenza biologica individuale a determinate classi terapeutiche di farmaci raccomandati per una terapia combinata (14). Nell'ultimo report della JNC solo i diuretici ed i beta-bloccanti sono considerati come terapie di primo livello per l'ipertensione lieve e moderata.

La scelta iniziale della terapia antipertensiva è molto importante perchè può essere efficace in circa il 50\% dei casi e, se non insorgono effetti collaterali indesiderati, sarà probabilmente portata avanti per anni. Invece, se il primo farmaco utilizzato manifesta fastidiosi effetti collaterali, la compliance del paziente tenderà a diminuire e ciò può comportare un insufficientie controllo farmacologico dell'ipertensione.

In sintesi, tutti i farmaci approvati negli USA per la cura dell'ipertensione sono efficaci nell'abbassare il livello della pressione arteriosa e quasi tutti sono inclusi nella Tabella 1 (cinque traiettorie esistenti) (tutti presentano un livello di efficienza biologica oscillante in un range compreso tra il 55\% e l'85\%) $(10,14,32)$. Tutti questi farmaci sono indicati per il trattamento dell'ipertensione lieve e moderata e, di conseguenza, tutti sono in grado di ridurre la pressione diastolica di almeno $10 \mathrm{~mm}$ di mercurio con un' analoga efficienza terapeutica. Come precedentemente accennato, solo i diuretici ed i betabloccanti hanno evidenziato, in trials clinici su larga scala, di essere in grado di ridurre la morbilità e la mortalità nei pazienti ipertesi. Tuttavia, gli ACE inibitori, i calcioantagonisti e gli alfa-bloccanti hanno comunque un effetto più favorevole su alcuni fattori di rischio cardiovascolare.

In particolari categorie di pazienti, un farmaco può essere più vantaggioso di altri. L'efficienza varia da una traiettoria all'altra quando l'ipertensione si presenta associata ad altre patologie (comorbilità). In questi casi, il JNC raccomanda terapie individualizzate per gruppi di pazienti come i diabetici, i soggetti con insufficienza renale o con artrite, gli obesi, i pazienti resistenti agli anti-ipertensivi e/o i pazienti la cui ipertensione dipende da fattori demografici (sesso, età, razza, etc.).

Anche quando la terapia farmacologica viene scelta attentamente per soddisfare le necessità individuali, la risposta può variare da paziente a paziente (32-33). Un'ulteriore considerazione da fare circa la scelta di un farmaco è, naturalmente, la disponibilità e la costo-efficacia dei vari medicinali nelle differenti aree geo- grafiche e nei diversi contesti socio-economici.

Partendo praticamente da ogni farmaco è possibile attuare un gran numero di modificazioni della struttura molecolare ed ogni nuova molecola ha la possibilità potenziale di aumentare l'attività biologica del prototipo. Tuttavia, la probabilità che si verifichino notevoli miglioramenti in una serie di analoghi strutturali è molto bassa. Questo è dovuto in parte al fatto che si conoscono molto pochi meccanismi d'azione molecolari mentre ci sono troppe teorie e ipotesi che tentano di spiegare come il farmaco agisca sull'organismo. Si è pure pensato che analoghi strutturali di un prototipo possano essere più potenti, meno tossici, possano avere un indice terapeutico più ampio o avere altre caratteristiche favorevoli (come consentire una migliore qualità di vita al paziente) (34).

Riepilogando, le modificazioni molecolari operate su un farmaco prototipo portano a sviluppare prodotti analoghi per il trattamento dell'ipertensione lieve e moderata, caratterizzati, nella grande maggioranza dei casi, da effetti terapeutici e reazioni avverse uguali o simili a quelle del prototipo. Ad esempio, nel confronto tra captopril e lisinopril, risulta più vantaggioso il secondo grazie alla somministrazione in un' unica dose giornaliera.

\section{TRAIETTORIE DI COMPETIZIONE TRA FARMACI ANTI-IPERTENSIVI}

La Tabella 1 mostra come ognuna delle differenti traiettorie sia integrata da diversi farmaci che condividono un principale meccanismo d'azione e come ogni farmaco sia associato a specifici brevetti che indicano la novità del prodotto. Come si può anche vedere nella Tabella 1, per quanto riguarda i farmaci vasodilatatori della traiettoria $T_{3}$, solo uno di quei prodotti (in forma orale) è commercializzato nel mercato messicano; perciò questa traiettoria non è stata ulteriormente tenuta in considerazione. La Tabella 2 indica i nomi commerciali degli ACE-inibitori, la sostanza chimica, il laboratorio e la data di introduzione di ognuna sul mercato messicano.

Le Figure 1 e 2 mostrano l'evoluzione di ogni traiettoria in termini di quota sia di unità sia di valore nel mercato messicano. Si è osservato che $T_{4}$ e $T_{5}$, costituite dalle più recenti classi di farmaci (calcioantagonisti e ACEinibitori), hanno conquistato un posto di rilievo nel mercato degli antipertensivi. Al contrario, $\mathrm{i}$ farmaci più vecchi di $\mathrm{T}_{1}$ e $\mathrm{T}_{2}$ (diuretici e beta-bloccanti) sono stati spiazzati o esclusi dall'adozione dei farmaci più nuovi. Come si può quindi vedere, le più recenti traiettorie $\left(\mathrm{T}_{4}\right.$ e $T_{5}$ ) hanno preso il posto di quelle più vecchie e meno costose $\left(\mathrm{T}_{1}\right.$ e $\left.\mathrm{T}_{2}\right)$. Come già ricordato, 
si è dato per scontato che tutte e cinque le traiettorie abbiano un'efficacia media del $50 \%$ come monoterapie ed in questo senso il processo di competizione tra le diverse traiettorie è descritto principalmente da altre fonti capaci di giustificare l'evoluzione di ciascuna traiettoria secondo differenti velocità di adozione dei nuovi farmaci.

Nelle Figure 1-3 e nelle Tabelle 3-7 viene rappresentato il processo di competizione tra le cinque traiettorie $\left(\mathrm{T}_{1}-\mathrm{T}_{5}\right)$. Qui si prendono in considerazione le quote di mercato degli antipertensivi, il numero di prescrizioni, le vendite annuali per ciascuna traiettoria, la quantità di compresse usate e il costo mensile di ogni farmaco all'interno delle cinque traiettorie. Per i farmaci $\mathrm{T}_{5}$ è stata elaborata un' analisi più dettagliata, dove si spiega ogni prodotto che compone questa traiettoria (Figura 4 e Tabella 7). In queste figure vengono rappresentate le quote di mercato per ognuno dei prodotti che compongono la traiettoria $\mathrm{T}_{5}$ e che dimostrano un 'efficienza simile nel trattamento dell'ipertensione. Si osserva che due sub-traiettorie dominano il mercato (Capoten ${ }^{\circledR}$ e Vasotec $\left.®\right)$ ). I restanti prodotti hanno mantenuto quote di mercato relativamente più basse nel periodo preso in esame.

Ad una prima analisi si nota che nessuna sub-traiettoria che compaia più tardi può spiazzare, a parità di efficienza, odiminuire drasticamente il peso delle sub-traiettorie dominanti. Le prime sub-traiettorie introdotte nel mercato mantengono le loro quote di mercato ed i livelli di vendita.

La competizione fra traiettorie e i differenti modelli di adozione originano da diverse fonti - l'apprendimento tramite l'uso, la produzione in economie di scala, le interrelazioni tecnologiche e le reti istituzionali - identificate nel mercato farmaceutico messicano dei prodotti per il trattamento dell'ipertesnione lieve e moderata.

\section{APPRENDERE DALL'USO NELLA TRAIETTORIA}

Più un farmaco viene usato, più aumenta la conoscenza che si può ottenere dal farmaco stesso. Partendo da queste conoscenze il farmaco può essere sviluppato e migliorato e nuovi prodotti possono essere introdotti sul mercato. Questo effetto si verifica per tutti gli antipertensivi, ma è interessante specialmente nel caso dei farmaci $\mathrm{T}_{5}$ : infatti, il numero di subtraiettorie ha avuto un grande sviluppo ed è ancora in crescita. C'era una lezione da imparare dal primo farmaco introdotto sul mercato ed i prodotti successivi sono il risultato della sua imitazione e delle conoscenze aggiuntive otte- nute (ad esempio, il caso di captopril e lisinopril nel mercato messicano).

\section{ECONOMIE DI SCALA IN PRODUZIONE PER LA TRAIETTORIA}

I costi diminuiscono grazie alla vendita del medicinale su larga scala. In questo caso, i limiti di tempo del brevetto consentono un processo dove il prodotto puòdiventare più competitivo nel prezzo se il suo utilizzo cresce. Ciò è interessante per tutti gli antipertensivi, ma in particolare per le sub-traiettorie di $\mathrm{T}_{5}$. Un maggior numero di farmaci generici possono essere ottenuti a prezzi inferiori. Come evidenziato nella Tabella 7, uno degli ACEinibitori più nuovi presente sul mercato messicano, Lotensin $®$ (benazepril), è il più caro, mentre, al contrario, uno dei più vecchi, il Glioten ${ }^{\circledR}$ (enalapril) è il meno costoso.

\section{INTERRELAZIONI FARMACOLOGICHE NELLE SUB-TRAIETTORIE}

Si tratta delle differenti caratteristiche associate ai farmaci che hanno la stessa efficacia (per esempio, il fatto che un farmaco richieda una sola somministrazione al giorno - come nel caso del lisinopril), tenendo bene a mente che tutte le traiettorie hanno lo stesso effetto desiderato: abbassare la pressione arteriosa nella stessa proporzione. E ancora, è necessario ricordare come ognuno dei farmaci delle diverse traiettorie presenta differenti reazioni avverse e costi differenti. Gli ACE-inibitori causano tosse in un'alta percentuale di pazienti, tra il $5 \% \mathrm{e}$ il $20 \%$. La tosse richiede un trattamento e quindi si ha un aumento di costi. I diuretici producono diuresi, come dice il nome, e l'uso prolungato può alterare l'equilibrio elettrolitico dell'organismo (bilancio di sale) e causare disturbi nutrizionali.

Un altro fattore importante riguarda il numero di somministrazioni che sarebbero necessarie. I pazienti non ospedalizzati possono dimenticarsi di prendere le medicine o possono assumerle non nel modo indicato dal medico e quindi ottenere risultati terapeutici insufficienti. La compliance può essere migliorata con farmaci da prendere una sola volta al giorno (ad esempio, lisinopril contro captopril). Inoltre, sul mercato esistono compresse con dosaggi irrazionali che non si riscontrano nella letteratura scientifica, fanno spendere una maggior quantità di soldi e richiedono un maggior numero di compresse per raggiungere la dose terapeutica. Tutto ciò peggiora la compliance.

Nessuno di questi fattori sembra aver determinato e spiegato il processo di competizione tra le sub-traiettorie. Sebbene si possano 


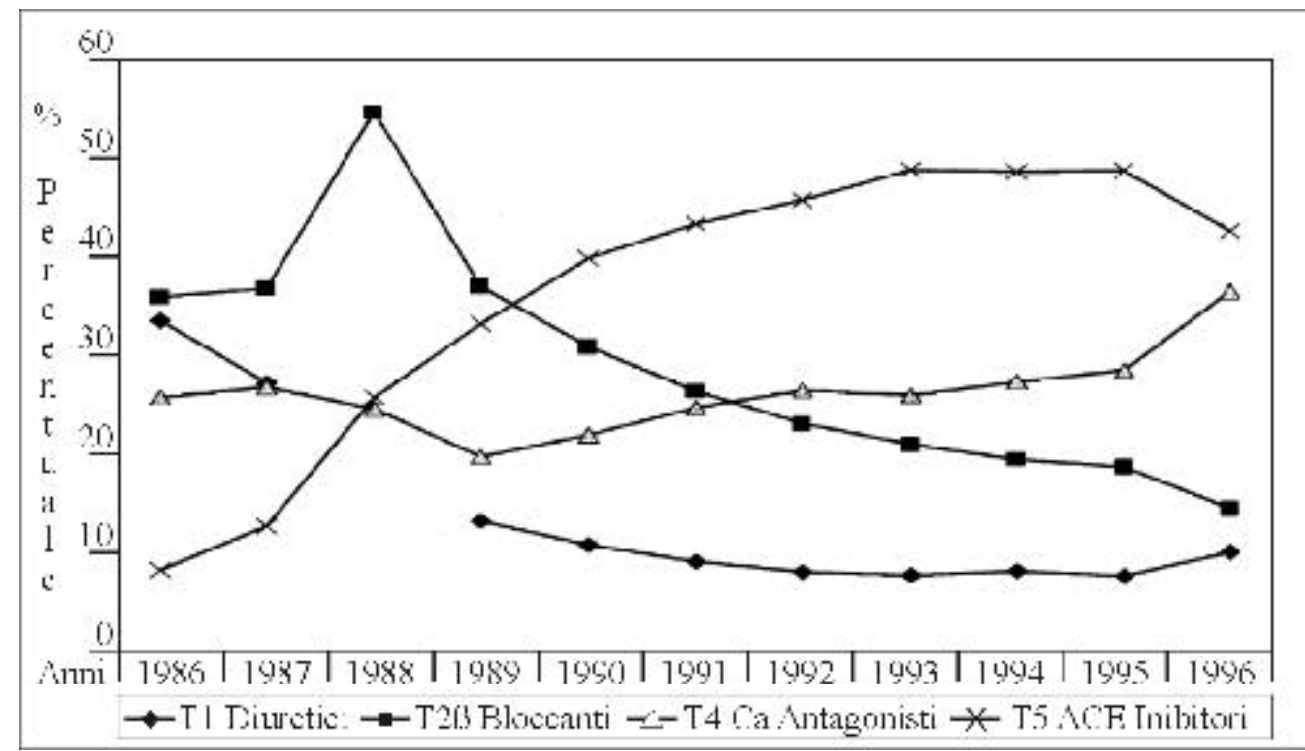

Fonte: International Marketing Services Mexico

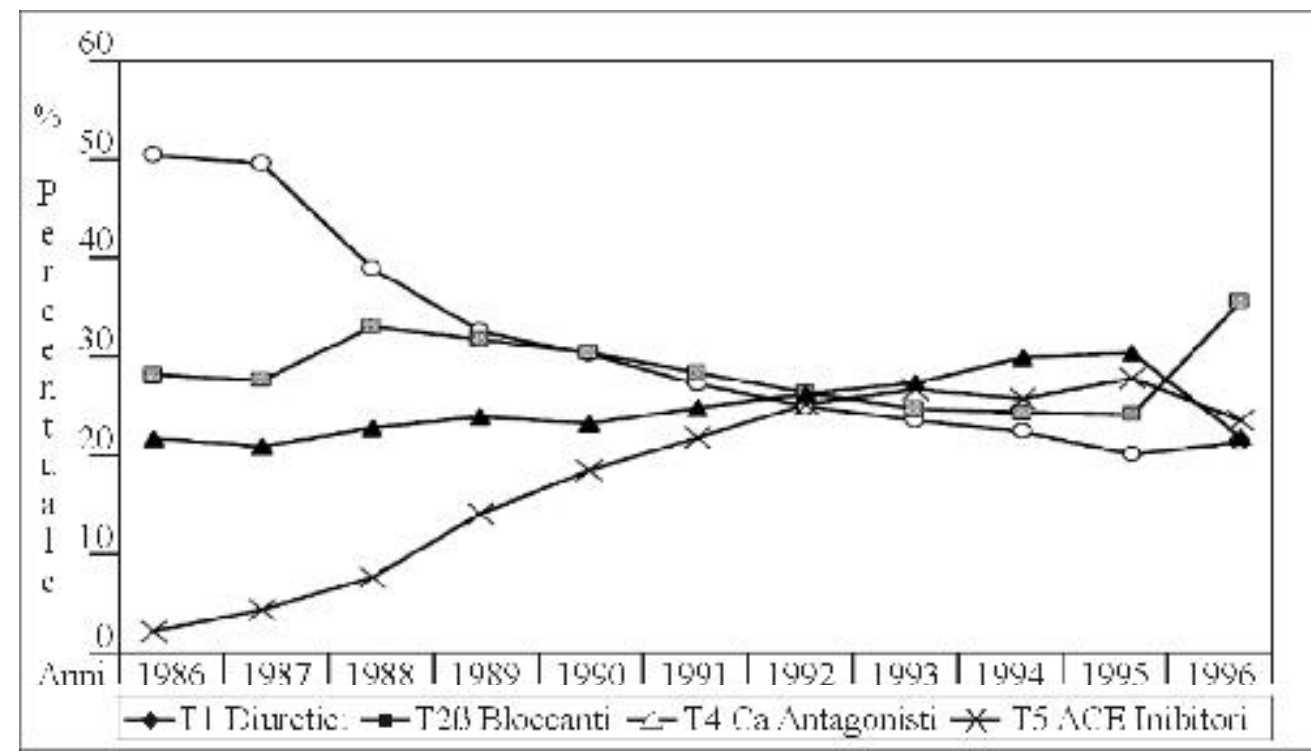

Fonte: International Marketing Services Mexico

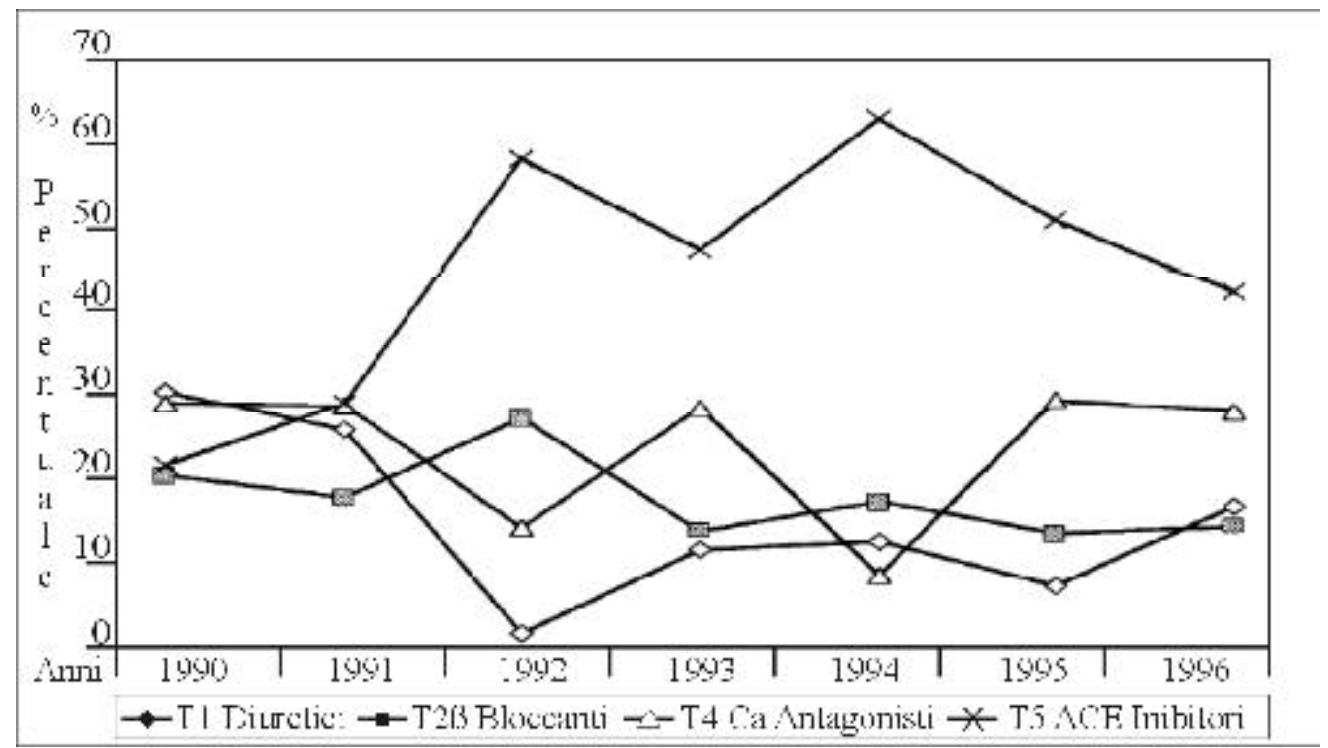

Figura 1

Evoluzione delle quote di mercato degli Agenti Antiipertensivi: percentuale del valore totale di mercato per le 4 categorie (traiettorie)

Figura 2

Evoluzione delle quote di mercato degli Agenti Antiipertensivi: percentuale del mercato totale in unità

Figura 3

Evoluzione delle prescrizioni dei farmaci contro l'ipertensione nel mercato messicano, 19901996: percentuale delle prescrizioni locali per l'ipertensione essenziale 
Tabella 3

Costo mensile, in Pesos messicani, del trattamento con diuretici $T_{1}, 1997$ (denominazioni cliniche marche commerciali)

Fonti: PLM Mexican Directory 1997; Retail Drugstore Prices, Mexico D.F.

osservare fenomeni di apprendimento derivan- promuovere incentivi e proporre l'adozione di te dall'uso, così come economie di scale e un farmaco. interrelazioni tecnologiche, due sub-traiettorie continuano ad essere dominanti nella competizione all'interno di $\mathrm{T}_{5}$.

C'è un chiaro effetto che deriva dal prestigio di essere il prodotto innovatore, effetto che si mantiene nel tempo. Un altro elemento fondamentale da spiegare è il motivo per cui i vantaggi dell' apprendimento derivante dall'uso, le economie di scala e le interrelazioni tecnologiche giustificano così debolmente la competizione tra le traiettorie in confronto al peso che assume la prevalente rete istituzionale del farmaco in ciascun paese.

La ragione per cui questo elemento continua a essere dominante e a rimanere stabile dipende dalla regolamentazione dei singoli paesi e dall' organizzazione della rete sanitaria, in termini di creazione di relazioni interpersonali per
Diversi fattori influenzano la quantità di risorse sanitarie consumate dal singolo individuo. Uno di questi fattori consiste nel fatto che chi acquista prodotti e servizi sanitari non è sempre il consumatore. L'acquirente può essere lo Stato o il Governo Federale, una compagnia di assicurazione sanitaria, una cooperativa, una società privata, un sindacato, un gruppo di consumatori, una famiglia o un individuo. Siccome i compratori pagano i prodotti ed i servizi, essi hanno una grande influenza sull'utilizzo di queste risorse. I vincoli di budget del singolo paziente o della famiglia possono limitare la quantità di risorse spendibili per la salute. I terzi paganti possono sia migliorare la capacità del paziente di pagare per l'assistenza sanitaria sia limitare i tipi e la quantità di assistenza che il paziente può ricevere. 


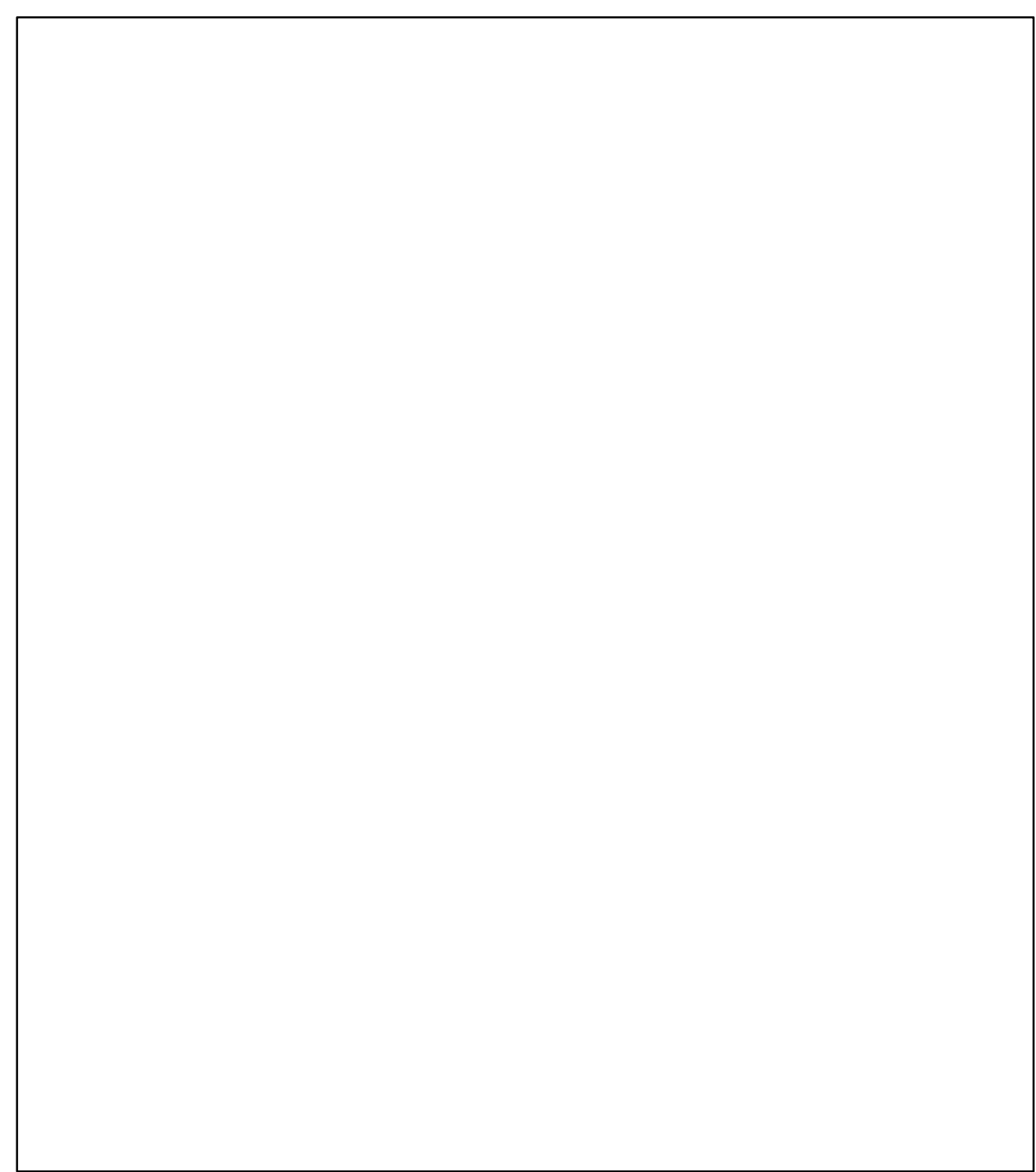

Tabella 4

Costo mensile, in Pesos messicani, del trattamento con bloccanti $T_{2}, 1997$ (nomi generici e marche)

Fonte: PLM Mexican Directory 1997; Retail Drugstore Prices, Mexico D.F.

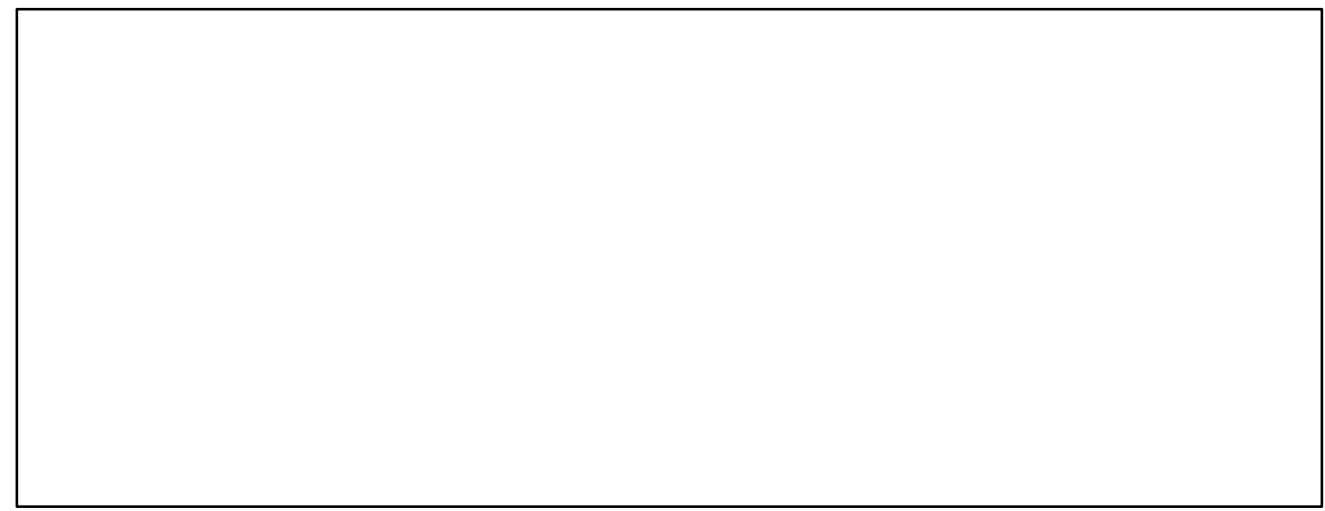

Tabella 5

Costo mensile, in Pesos messicani, del trattamento con vasodilatatori (generici e marche)

Fonti: PLM Mexico, 1997; Retail Drugstore Prices

* L'unica forma orale di questa classe disponibile in Messico 
Tabella 6

Costo mensile, in Pesos messicani, del trattamento con antagonisti del calcio $\mathrm{T}_{4}$ (denominazioni cliniche e marche) 1997

\section{Figura 4}

Percentuale delle quote di mercato dei principali ACE inibitori $\left(\mathrm{T}_{5}\right)$ indotti nel mercato messicano; sviluppo finanziario (per marche)

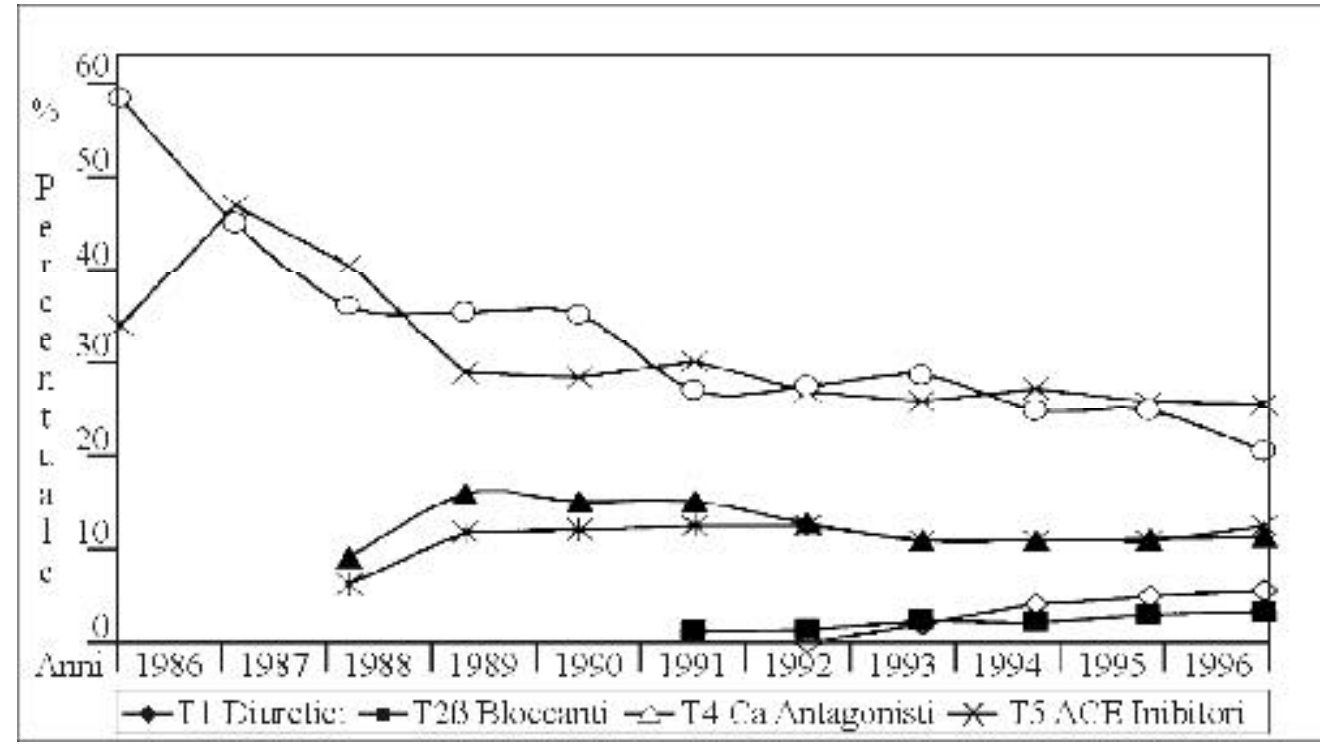

Fonte: International Marketing Services Mexico 


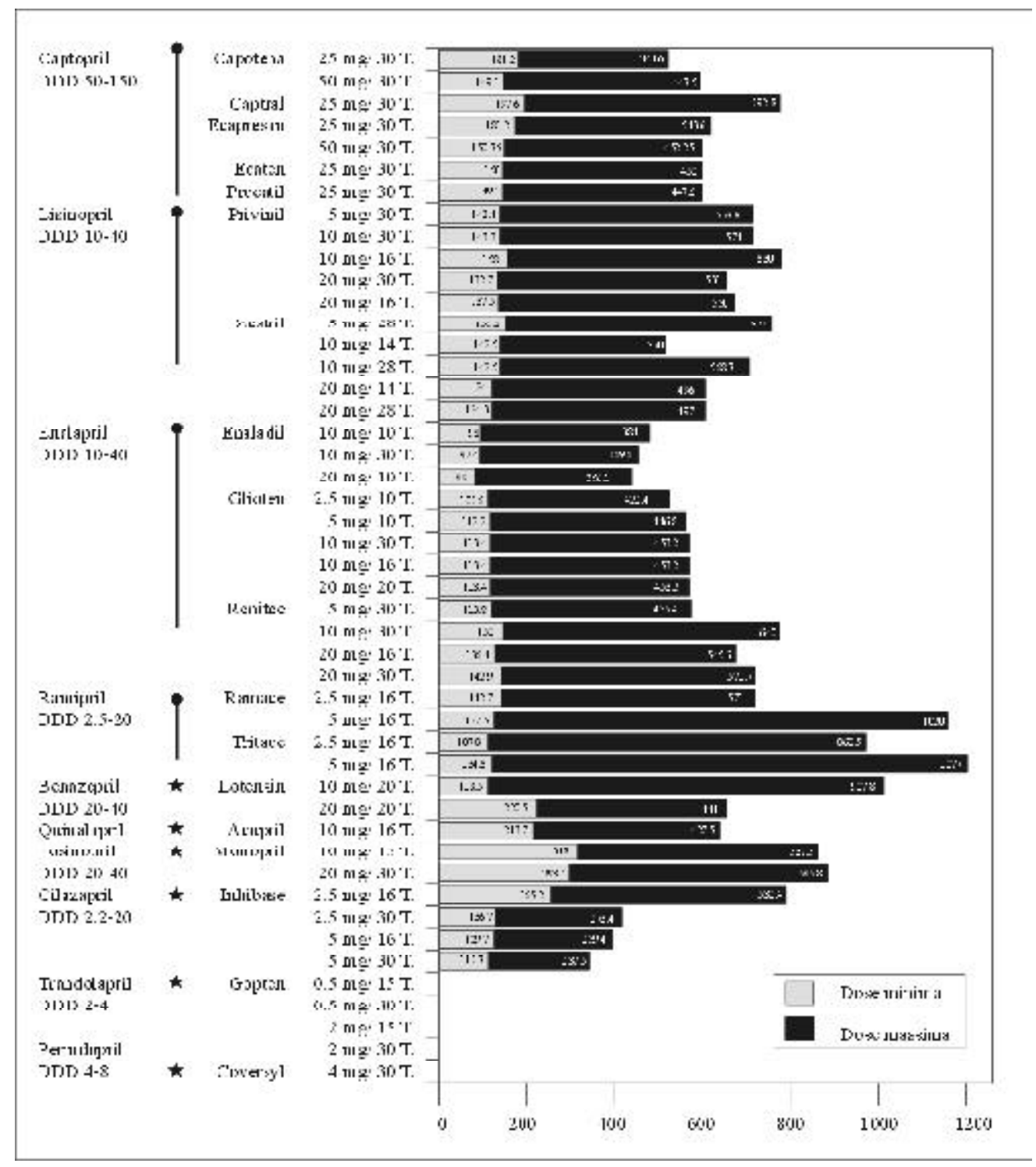

Tabella 7

Costo mensile, in Pesos messicani, del trattamento ACE inibitori (farmaci generici e marchi) 1997

Fonti: PLM Mexican Directory 1997; Retail Drugstore Prices, Mexico D.F.

Un fattore che influenza il consumo di risorse sanitarie è rappresentato dalla prescrizione di servizi e prodotti da parte del medico. Sebbene il paziente sia il primo fruitore del sistema sanitario, è il medico il primo decisore nel sistema sanitario. Le decisioni del medico possono influenzare la qualità, la quantità, il tipo e il costo dei prodotti e dei servizi sanitari utilizzati dai pazienti.

In Messico le farmacie vendono medicinali con o senza prescrizione medica, e non hanno un registro delle ricette, tranne per alcuni farmaci controllati. Invece, secondo la legge, per la vendita al pubblico di farmaci che contengono composti psicotropi, tipo i narcotici, occorre avere uno speciale registro delle prescrizioni fornito dal Ministero della Sanità ai professionisti. Questa situazione è la ragione principale dell'automedicazione. Il personale che vende medicinali in farmacia non è affatto diverso dal venditore di scarpe o TV. In Messico il farmaci- sta non viene considerato un membro del sistema sanitario e quindi non ha alcuna funzione di educazione sul paziente. La farmacia clinica non è sviluppata nel paese, nonostante siano stati compiuti alcuni sforzi isolati per organizzarla.

Il Sistema Sanitario Nazionale (SNS) per legge deve fornire i medicinali, sostenendone le spese. Negli ultimi anni, tuttavia, la fornitura di farmaci è stata insufficiente e non ha coperto tutti i medicinali inclusi nel prontuario di base, e neanche tutti i pazienti. Molti medici del SNS visitano anche i pazienti in studi privati, dove ricevono la visita degli informatori delle case farmaceutiche.

Inoltre c'è anche molta diffidenza nei confronti dei farmaci generici, perché quel tipo di mercato non si è ancora sviluppato e la gente preferisce la prescrizione di farmaci di marca. Nel passato vi sono stati problemi per quanto riguarda la qualità dei farmaci generici acqui- 
stati dal governo attraverso canali di vendita consolidati.

Questi fattori vanno a incrementare il numero di consumatori che si rivolgono alle farmacie private e promuove anche origine un sistema orientato all'automedicazione e un più ampio numero esternalità di rete. Man mano che il numero dei consumatori cresce, aumenta anche la consapevolezza che esistono altri utenti. Si verificherà quindi una maggiore adozione di un determinato farmaco, ma anche più reazioni avverse ed effetti collaterali: e l'automedicazione non permette di controllare queste reazioni avverse e secondarie. Il sistema di farmacovigilanza quindi non funziona. L'automedicazione fa aumentare il volume di vendita dei farmaci, ma questo non è necessariamente un miglior indicatore di salute. Diminuisce la spesa per visite mediche, ma non necessariamente quella per ospedalizzazione (le reazioni avverse possono causare malattie o morte).

Il ruolo delle compagnie di assicurazione e dello Stato nel promuovere l'adozione di farmaci assume un peso maggiore, collegando la rete sanitaria con l'atto prescrittivo. Quando la rete non è collegata alle compagnie di assicurazione e allo Stato, le case farmaceutiche hanno maggiore spazio per influenzare la prescrizione mediante azioni di marketing.

E' evidente che tra due opposti modelli vi possono essere soluzioni intermedie. Per esempio, potrebbe darsi un sistema con una forte presenza di automedicazioni e una scarsa influenza da parte dello Stato, nel quale le compagnie di assicurazione assumono il ruolo di principale terzo pagante. Oppure si può ipotizzare che il sistema sia strettamente controllato dallo Stato o sotto la costante pressione delle compagnie di assicurazione. In tutti questi casi, vi sono motivi molto differenti per ricorrere ad una traiettoria piuttosto che ad un'altra.

In Messico esiste una forte quota di automedicazione, con una scarsa regolamentazione in materia di salute pubblica ed una debole pressione dello Stato sull'atto prescrittivo e sul controllo delle prescrizioni. Anche il controllo sulla legalità della presenza del farmaceutico in farmacia e nel sistema sanitario è piuttosto debole. Tutti questi elementi spiegano la forte presenza strategica delle case farmaceutiche per influenzare l'introduzione dei loro farmaci.

Una scarsa presenza dello dello Stato come fonte di informazioni sulle alternative farmacologiche, una scarsa presenza delle compagnie di assicurazione nella dei farmaci in base ai costi ed all'efficacia, una forte tendenza all'automedicazione, la presenza nel sistema sanitario di operatori non esperti (gli operatori commerciali non conoscono i prodotti né i loro processi terapeutici) e di forti strategie di marketing delle case farmaceutiche sono tutti fattori che contribuiscono a spiegare le dinamiche di competizione (e l'adozione da parte dei consumatori) delle sub-traiettorie considerate.

\section{CONCLUSIONI}

Il concetto di traiettorie e l'analisi dei processi competitivi che si sviluppano tra loro, mai ancora applicati in precedenza al mercato farmaceutico, offrono ad organizzazioni assicurative, agenzie sanitarie governative, gruppi di fornitori e produttori, un nuovo entusiasmante strumento per analizzare tendenze ed eventi secondo una struttura logica consistente ed organizzata. L'apprendimento correlato all'uso, le interrelazioni farmacologiche e la rete istituzionale del farmaco hanno costituiscono il principale strumento di analisi usato per spiegare le dinamiche di competizione tra le differenti traiettorie prese in considerazione.

Questo tipo di tecnica consente al consumatore di valutare il livello di soddisfazione nei confronti delle opzioni terapeutiche esistenti. Per esempio, se un dato farmaco incontra un vasto gradimento, potremmo aspettarci di osservare che nella maggior parte dei trattamenti si utilizza quel farmaco. Dall'altra parte, se riscontriamo che vengono usati molti farmaci differenti, questo indica che nessuno dei trattamenti esistenti incontra la piena soddisfazione degli utenti. Questo fatto indica ai produttori che in questo settore esiste un mercato non ancora raggiunto e suggerisce alle assicurazione di porsi la domanda se usare qualcuno dei farmaci non totalmente soddisfacienti sia meglio di non usare alcun farmaco. L'opzione di non usare farmaci potrebbe essere meno costosa e sicuramente provocherà meno effetti collaterali.

I sociologhi e gli esperti di ricerche di mercato apprezzeranno le analisi di traiettorie nel momento in cui saranno loro di aiuto per classificare e descrivere i comportamenti, specialmente nell'ambito dell'adozione di nuovi prodotti. Con questo strumento si può osservare quanto i prescrittori siano affezionati a un prodotto già esistente (fedeltà) e quale grado di miglioramento sia necessario per modificare questa fedeltà. Gli esperti di marketing potrebbero essere in grado di determinare se un nuovo prodotto, frutto di miglioramenti, per diventare un successo commerciale richieda di avere una maggiore efficacia, minori effetti collaterali o, per esempio, un differente sapore.

Esiste probabilmente un vasto utilizzo potenziale non ancora sfruttato dell'analisi di tra- 
iettoria da parte di epidemiologi e di pianificatori sanitari. Modelli di prevalenza di malattia potrebbero essere monitorati usando il consumo dei farmaci come una misura grezza. Questo tipo di profili di consumo potrebbero essere più affidabili rispetto ai dati di autoriportati o indicati dai fornitori.

Infine, sarebbe un utile esercizio confrontare i risultati ottenuti con questo metodo basato sul concetto di traiettoria con quelli ottenuti da uno studio che utilizzi il costrutto teorico convenzionale proposto da Everett Rogers quasi 40 anni fa per analizzare la diffusione delle innovazioni (35). E molto probabile che una qualche integrazione di queste due visioni/ paradigmi possa fornire risposte a questioni che, separatamente, nessuno dei due sistemi è in grado di risolvere. Nel modello di Rogers, si trova un gruppo di consumatori etichettato come "pionieri" nell' adottare un prodotto, un altro gruppo etichettao come come "seguaci", e un altro ancora etichettato come consumatori "tardivi”. Questi gruppi di consumatori possiedono diversi tratti di personalità o valutano aspetti differenti?

Oggigiorno vi sono solo poche preziose spiegazioni per alcuni aspetti irrazionali del comportamento prescrittivo che è così dispendioso e potenzialmente dannoso. Forse l'aggiunta dell'analisi basata sulle traiettorie costituirà la chiave per nuove illuminanti interpretazioni.

\section{BIBLIOGRAFIA}

1. David PA. Clio and the economics of QWERTY. Am Econ Rev 1985; 75:332-7.

2. Dosi G. Opportunities, incentives and the collective patterns of technological change. Econ J 1997;107:1530-47.

3. Arthur WB. Competing technologies, increasing returns and lock-in by historical events. Econ J 1989;99:116-31.

4. Cimoli M, Dosi G. Technological paradigms, patterns of learning and development: an introductory roadmap. J. Evolutionary Econ 1995;5(3):243-68.

5. Cimoli M. Della Giusta M. The nature of technological change and its main implications on national and local systems of innovation. In: Learning, innovation and urban evolution. Batten D, Bertuglia CS, Martellato D, Occell S. eds. New York: Springer Verlag, 1999.

6. Arthur WB. Competing technologies: an overview. In: Technical change and economic theory. Dosi G. Nelson R. Soete L. eds. London: Pinter, 1988;591-607.

7. Rosenberg N. Inside the black box: technology and economics. Cambridge, MA: Cambridge University Press, 1982.

8. Frankel M. Obsolescence and technological change in a maturing economy. Am Econ Rev 1955;45:296-319.

9. Katz M. Shapiro C. Network externalities, competition and compatibility. Am Econ Rev 1985;75:424-11.

10. Oates JA. Antihypertensive agents and the drug therapy of hypertension. In: Goodman \& Gilman's the pharmacological basis of therapeutics. 9th ed. Hardman JG, Limbird LE, eds. New York: McGraw-Hill;1996:780897.

11. Nichol MB. Margolies JE. Gill MA. Treatment approaches to hypertension. Ann Pharmacother 1997;31(2):154-9.

12. Secretaria de Salud de Mexico. Encuesta Nacional de Enfermedades Cronicas. Secretaria de Salud. Mexico D.F., Mexico, 1993.

13. The Merck index. 11th ed. Rahway, NJ: Merck, 1989.

14. Kirby R. Hypertension: therapeutic advances and market opportunities. Scrip Rep 1997;(Sept 4):1-175.

15. Hollenberg NK. Treatment of hypertension: the place of angiotensin-converting enzyme inhibitors in the nineties. J. Cardiovac Pharmacol 1992;20(Suppl 10): S29-S32. 
16. Gross TP. Wise RP, Knapp DE. Antihypertensive drug use: trends in the United States from 1973 to 1985. Hypertension 1989;13 (Suppl I): I113-I118.

17. Fleckenstein A. History of calcium antagonists. Circ Res 1983; 52(Suppl I): 3-16.

18. Scrip Magazine 1997;(Jan):45-6.

19. Dosi G. Sources, procedures, and microeconomic effects of innovation. J. Econ Lit 1988;26:1120-71.

20. David PA. Path-dependence and predictability in dynamics system with local network externalities: a paradigm for historical economics. In: Technology and the wealth of nations: the dynamics of constructed advantage. Foray D. Freeman C. eds. London: Pinter, 1993. 